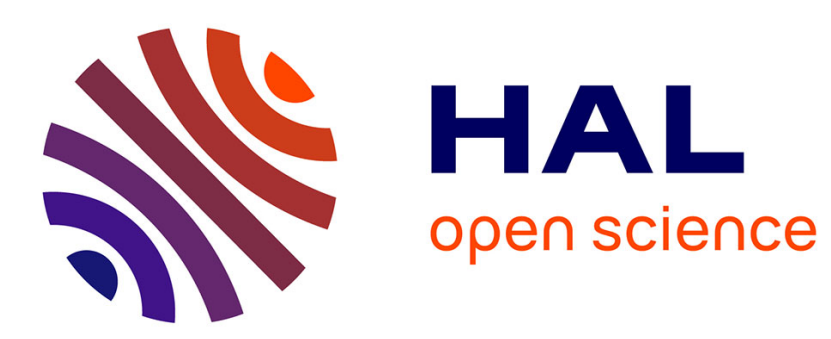

\title{
The Correlation framework: bridging the gap between modeling and analysis for 3D woven composites
} Arturo Mendoza, Julien Schneider, Estelle Parra, Stéphane Roux

\section{To cite this version:}

Arturo Mendoza, Julien Schneider, Estelle Parra, Stéphane Roux. The Correlation framework: bridging the gap between modeling and analysis for 3D woven composites. Composite Structures, 2019, pp.111468. 10.1016/j.compstruct.2019.111468 . hal-02296558

\section{HAL Id: hal-02296558 https://hal.science/hal-02296558}

Submitted on 25 Sep 2019

HAL is a multi-disciplinary open access archive for the deposit and dissemination of scientific research documents, whether they are published or not. The documents may come from teaching and research institutions in France or abroad, or from public or private research centers.
L'archive ouverte pluridisciplinaire HAL, est destinée au dépôt et à la diffusion de documents scientifiques de niveau recherche, publiés ou non, émanant des établissements d'enseignement et de recherche français ou étrangers, des laboratoires publics ou privés. 


\title{
The Correlation framework: bridging the gap between modeling and analysis for 3D woven composites
}

\author{
Arturo Mendoza ${ }^{\mathrm{a}, \mathrm{b}}$, Julien Schneider $^{\mathrm{c}}$, Estelle Parra ${ }^{\mathrm{b}}$, Stéphane Roux ${ }^{\mathrm{a}}$ \\ ${ }^{a}$ LMT (ENS Paris-Saclay/CNRS/Univ. Paris-Saclay), \\ 61 Avenue du Président Wilson, 94235 Cachan, France \\ ${ }^{b}$ Safran Tech, Rue des Jeunes Bois, 78772 Magny les Hameaux, France \\ ${ }^{c}$ Safran Aircraft Engines, Rond Point Réné Ravaud - Réau, 77550 Moissy-Cramayel, France
}

\begin{abstract}
A large variety of different descriptors can be employed for describing 3D woven composites. Each of them is targeted to a specific application, ranging from design, weaving, molding, impregnation down to non-destructive testing, imaging and numerical modeling of the "actual" micro-structure. In order to relate these different representations, it is proposed herewith to rely on the inherent weaving pattern as an intrinsic common feature, and to resort to images as a common language to guaranty the continuity of information. To connect these 3D images (either "real" or synthesized), Digital Volume Correlation (DVC) is called for in order to exploit the conservation of topology. A complete test scenario is devised in which different manufactured woven samples are compared to the theoretical textile arrangement. The results confirm the effectiveness of the method.
\end{abstract}

Keywords: woven composites, digital volume correlation

\section{Introduction}

The ever-increasing interest in composite materials has generated a high demand for new custom tools. They include tailored modeling strategies [1], proper characterization methods [2], accurate simulations [3] and adapted non-

\footnotetext{
*Corresponding author

Email address: arturo.mendoza-quispe@safrangroup.com (Arturo Mendoza)
} 
destructive testing (NDT) techniques based on high-resolution X-ray computed tomography (micro-CT) images [4].

However, these analyses tend to be developed and carried out independently of each other. Naturally, this occurs mainly due to the fact that each procedure uses a different "textile descriptor". Indeed, each of these descriptors conceives the woven composite in a manner that better suits the target analysis. For example, some statistical descriptors extracted from CT images may be best suited for NDT (such as the structure tensor [5]). On the contrary, textile simulations may be best served by Finite Element (FE) [6] meshes or even "virtual models" (CAD) that represent the structure of the textile [7]. As a result, the analyses cannot (completely) benefit from the results of each other, which also limits the confrontation between real (experimental) data and modeling (or numerical simulations) [8].

Nonetheless, all these textile descriptors share a common characteristic: the weaving pattern. Such pattern defines the relative position of yarns (reinforcement) that is maintained by the polymer resin (matrix). This definition is intrinsic to the material and always present, even if the target analysis may not require it (e.g., statistical descriptors). It is noteworthy that this always-present feature will not be "identical" in all the descriptors. For example, the yarn positions (in the three-dimensional space) of a given textile will differ between a "theoretical" representation versus a "real" one, or even simply a "realistic" one [9]. Thus, relating any pair of descriptors only requires the identification of these differences.

Moreover, these descriptors can also be expressed under a common language: that of an image, that is they can generate images looking "alike". Again, this similarity comes from the material itself; in particular from the two constitutive phases of the composite.

As formulated, the inherent problem is actually quite close to that encountered in Digital Volume Correlation (DVC) [10, 11]: to retrieve the displacement field relating pairs of configurations. As such, it is possible to consider the material as in a so-called deformed or "wrapped" configuration with respect to a 
reference one [12]. These configurations can be embodied by any of the considered textile descriptors. Furthermore, by bridging the gap [13, 14] between these elements, all the associated analyses are immediately comparable. In like manner, this allows interpreting the various results obtained from these different analyses from a unique standpoint: that of their underlying topology. This notion is at the origin of the so-called unique topological descriptor.

The advantages of such an approach are many. First, a continuous digital information chain can be constructed. As such, there is no need for storing redundant information or coming up (missing) information discarded by previous processes, encouraging a more conscious use of the data and capitalizing on previous (potentially costly) efforts. Second, it can be used as a tool for rearranging the data into more convenient representations. For example, aligning the warp and weft orientations with the image axes should aid in the task of "correcting" warped yarn structures. In particular, two warping modes are well known in this field: (i) "high shear angles" which result in non-orthogonal warp and weft orientations, and (ii) "warped columns" which result in yarn layers with relative in-plane offset. Such preprocessing can have a profound impact on the steps that may be followed, as well as for improving the data exploration. Third, since this framework is relative by nature, any discrepancy is automatically flagged (i.e., NDT). As such, weaving anomalies (e.g., missing yarns or loops) can be identified without developing custom or additional systems, or even actively seeking them.

Finally, given that the current study focuses on the weaving pattern, the meso-scale [15] is chosen henceforth. The concerned descriptors (and a proposed classification system) will be detailed in Section 2. The registration and discretization procedures will be discussed in Sections 3 and 4 respectively. The method is then tested on diverse sets of textile descriptors, presented in Section 5. Using these descriptors, a numerical test is performed between two descriptors of the same type. Next, a relatively simple but real scenario is studied between descriptors of different nature. And finally, a more complex case is studied by integrating the different aspects of previous tests. These results are 
shown in Section 6.

\section{Overview of textile descriptors}

As discussed earlier, there exist many types of textile descriptors. One can attribute this plurality to the hierarchical nature of the material, which leads to a similar "organization" of descriptors. A representation of such often used descriptors combined into one reference frame is provided in figure 1, such is one of the outcomes of the present study. The following is a general overview of some of the descriptors that are of interest for the present study.

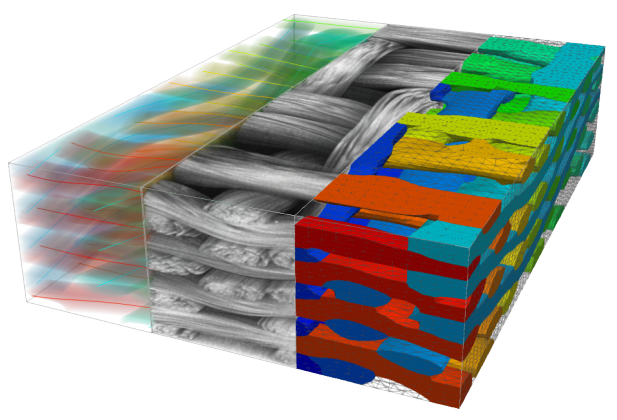

Figure 1: Multiple textile descriptors for the same textile architecture.

\subsection{Topological encoding}

The encoding of the structure of a 3D weave describes the relative positions of all yarns. It prescribes a topology, but does not consider any geometrical features (e.g., distance between yarns).

Let us consider a warp-interlaced 3D weave, such as the one shown in figure 2. Here, the warp yarns undulate around the weft yarns, while the latter remain "straight" at fixed positions. Then, the topological description of a multi-layered composite is based on the warp yarn paths. These paths are described as a sequence of intersection codes.

This description is best organized as a matrix, as shown in figure 3. Each matrix entry holds the value pertaining to an intersection between a given warp 


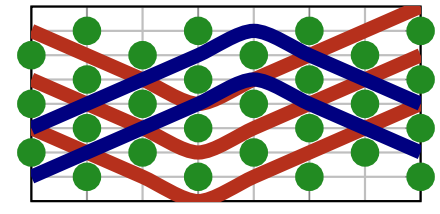

(a) Horizontal warp orientation

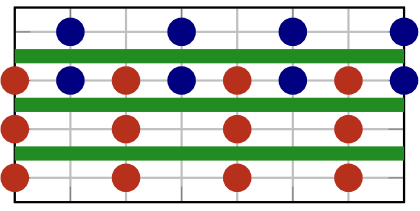

(b) Horizontal weft orientation

Figure 2: Topological encoding of warp-interlaced composite: while the green ( $\square$ ) weft yarns are modeled straight along their orientation, the red ( $\square$ ) and blue (匹) warp yarns undulate in different planes

yarn and a given weft row. While it is possible to list all possible intersections (between all warp yarns against all weft yarns), a more succinct matrix can be obtained if only the "pertinent" intersections are listed.

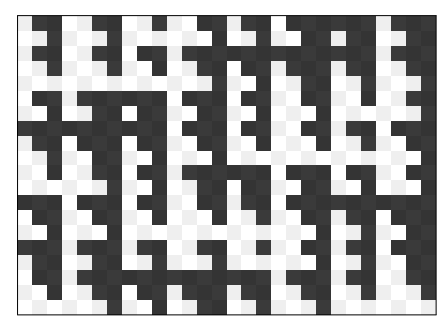

(a) Using binary codes

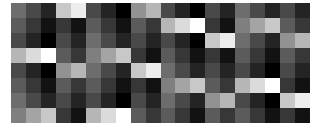

(b) Using level codes

Figure 3: Topological encoding in the form of a matrix

The former description holds more similarity to the traditional checkerboard pattern used for $2 \mathrm{D}$ composites, in which the path of the warp yarn is described as being either above or below the corresponding weft row. This limits the possible matrix values to zero or one, as shown in figure 3a On the other hand, the latter employs intersection level codes, as shown in figure 3b, that identify the weft layer situated above the warp yarn at a given intersection. For completeness, the number of warp planes and the number of weft columns need to be specified. 


\subsection{Geometrical encoding}

A geometrical descriptor [7] builds upon a topological one by placing the yarns in the three-dimensional space. It places the yarns via their centerlines, also known as neutral fibers. This one-dimensional curve is defined in the threedimensional space using a series of control points (3D coordinates). These can be limited to a reduced set of "master" points and employ interpolation functions in-between. Afterwards, the surface of the yarn is defined by sweeping a twodimensional shape along the length of the yarn. This cross-section can take the form of a simple (oriented) parametric curve (e.g., an ellipse) or a list of points that define a more complex shape.

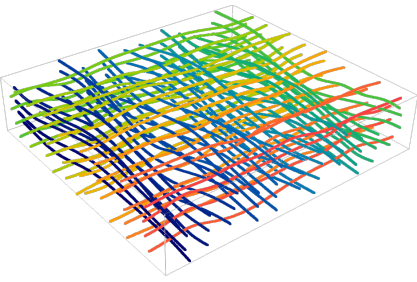

(a) Yarn neutral fibers

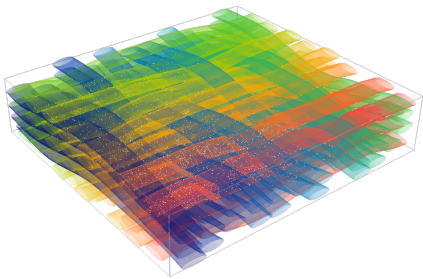

(b) Yarn cross-sections

Figure 4: Geometrical encoding for a sample textile

It should be noted that, thanks to the given cross-sections, the yarns can be considered as "solid" entities. For such a reason, if the target analysis requires it, the possible yarn inter-penetrations should be dealt with. This involves adapting the yarn centerlines as well as the yarn cross-sections. Multiple approaches have been conceived for dealing with this issue. These include textile geometrical modeling approaches [16], mechanical simulations [17], as well as image-based techniques [18].

\subsection{Finite Element encoding}

This descriptor builds upon the previous ones by adding some physical properties, that is, by endowing each point of the neutral fiber with some material properties. These include local bending stiffness, frictional and tensile behavior, 
fibrous content, fiber orientation, amongst others [19, 20]. This information is then employed to construct the FE meshes.

In general, a mesh is a discretization of a continuous spatial domain into a discrete one. As such, any volume can be subdivided into smaller (and simpler) elements that can be modeled using simple equations. Hence, the volume in question will vary according to the target analysis. Given that there are two phases in the material, it is possible to mesh only the yarns or to mesh the yarns and the resin.

Furthermore, there exists multiple paradigms for designing the mesh, notably a "conformal" approach and a homogenized one, as shown in figure 5. While the former aims at presciently meshing the yarn-resin (or yarn-air) and yarn-yarn interfaces [18], the latter allows more flexibility by considering "mixed" elements that account for both phases (e.g., the voxel-FE paradigm [21]).

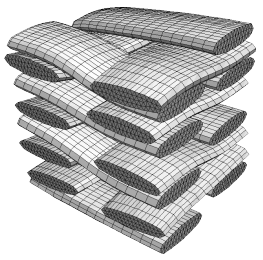

(a)

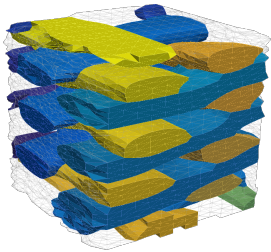

(b)

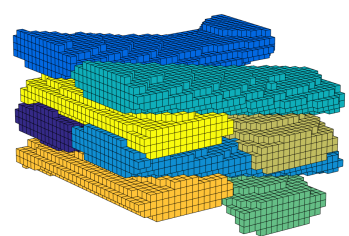

(c)

Figure 5: Multiple Finite Element encodings for a sample textile: (a) only yarns or (b)-(c) both phases can be meshed, using (a)-(b) pyramid or (c) cube FE element types

\section{Registration method}

This section will detail the elements required for the development of the socalled "correlation framework". Such approach will allow the relative analysis of different textile descriptors using DVC. Given that these descriptors may not have a rich texture (i.e., binary images) a strategy will be presented so as to overcome this issue. Furthermore, the scope of this framework includes 
descriptors of different types and sources. Hence the need for a "translation" step that provides an intermediate representation common to all descriptors.

\subsection{Correlation procedure}

Digital Volume Correlation [22] is a widely used technique for measuring the internal displacement field between volume pairs, generally obtained from tomography. For a reference image $f(\boldsymbol{x})$ and a test image $g(\boldsymbol{x})$, DVC minimizes the $L_{2}$ norm of residuals

$$
\eta=f(\boldsymbol{x})-g(\boldsymbol{x}+\boldsymbol{u}(\boldsymbol{x})) \cdot v_{1}(\boldsymbol{x})-v_{0}(\boldsymbol{x})
$$

with the optimal displacement field $\boldsymbol{u}(\boldsymbol{x})$ and intensity level corrections $v_{0}(\boldsymbol{x})$ and $v_{1}(\boldsymbol{x})$. The continuous transformation defined by $\boldsymbol{u}(\boldsymbol{x})$ implicitly includes an assumption of invariant topology between the analyzed volumes (i.e., the yarns are assumed to be organized in the same fashion). On the other hand, the corrections $v_{0}(\boldsymbol{x})$ and $v_{1}(\boldsymbol{x})$ explain all phenomena alien to such a hypothesis (e.g., tomographic reconstruction artifacts).

Given that the problem is ill-posed, well-posedness can be achieved and conditioning may be tuned when the displacement and correction fields are restricted to a space of low dimension. A global variational formulation [11] is used to determine the unknown fields $\boldsymbol{u}(\boldsymbol{x}), v_{0}(\boldsymbol{x})$ and $v_{1}(\boldsymbol{x})$. As such, any field $w(\boldsymbol{x})$ will be expressed as a function of the degrees of freedom $\{\boldsymbol{a}\}$ associated with it:

$$
w(\boldsymbol{x}) \approx \sum a_{i} \phi_{i}(\boldsymbol{x})
$$

This decomposition is used to describe the fields $\boldsymbol{u}(\boldsymbol{x}), v_{0}(\boldsymbol{x})$ and $v_{1}(\boldsymbol{x})$. A convenient choice for the kinematic basis $\phi_{i}(\boldsymbol{x})$ is one provided by the Finite Element (FE) method [6]. It should be noted that each of these fields could be decomposed differently (e.g., different meshes for each one) but, for the sake of simplicity, they will be expressed under the same formalism. As such, all the degrees of freedom associated to the three fields of interest can be grouped into a single vector of parameters $\{\boldsymbol{a}\}$. 
The overall solution is given by the minimization of the squared $L_{2}$-norm of the residuals over the entire region of interest. Such optimization problem can be solved with classical iterative Newton-Raphson routine. This leads to the following linear system

$$
[\boldsymbol{M}]\{\delta \boldsymbol{a}\}=\{\boldsymbol{b}\}
$$

with the "update" vector $\{\delta \boldsymbol{a}\}$, the (positive) stiffness matrix $\boldsymbol{M}$ and the vector $b$

$$
\begin{aligned}
M_{i j} & =\left\langle\boldsymbol{\psi}_{i}, \boldsymbol{\psi}_{j}\right\rangle \\
b_{i} & =\left\langle\boldsymbol{\psi}_{i}, \eta\right\rangle
\end{aligned}
$$

where $\langle\cdot, \cdot\rangle$ denotes the inner product (i.e., a contraction over $\boldsymbol{x}$ ), and the field $\boldsymbol{\psi}_{i}(\boldsymbol{x})$

$$
\psi(\boldsymbol{x})=\phi(\boldsymbol{x}) \otimes s(\boldsymbol{x})
$$

that translates to

$$
\boldsymbol{\psi}_{i}(\boldsymbol{x})=\phi_{i_{1}}(\boldsymbol{x}) \boldsymbol{s}_{i_{2}}(\boldsymbol{x})
$$

with the "sensitivity" field $s_{i_{2}}$

$$
s_{i_{2}}=\left(\nabla_{x} f, \nabla_{y} f, \nabla_{z} f, f, 1\right)
$$

and the "super-index" $i=\left(i_{1}, i_{2}\right)$ that relates $i_{1} \in\left[1, N_{n}\right]$, which points to a node (with $N_{n}$ the number of nodes), and $i_{2} \in[1,5]$, which denotes the associated degree of freedom.

\subsection{Longer correlation length}

One of the advantages of "classical" DVC is its high measurement accuracy [11]. Such extreme sensitivity to displacements is a result of the (wellcontrasted) textures being analyzed. In fact, such rich textures translate to rich image gradients varying in all directions.

However, the current context calls for images whose texture may be extremely poor. Any binary image will only contain non-zero gradients on the 
boundaries of the individual objects. Additionally, such boundaries are extremely localized near the phase boundaries. Thus a direct implementation of the DVC strategy will fail to converge unless the initialization is extremely close to the solution. For such reason, a relaxation scheme that will spread the gradients produced by the boundaries is proposed. This relaxation will progressively increase the correlation length and thus guide DVC to a good solution. It can be achieved by means of a Gaussian kernel with varying radius that is defined with respect to the largest displacement sought.

An example of the procedure is shown in figure 6. Here, a tomographic image of a composite part is recursively filtered by the same Gaussian kernel (with radius $2 \mathrm{px}$ ). The widened boundaries extend the effective correlation length in those regions.

Such procedure hints to the use of a multiresolution isotropic approach [23]. It consists in the use of a Gaussian pyramid on a isotropic version of the input images. The image pyramid allows to transition from a coarse description of the problem towards a finer one. This is aided by the subsampling required to transition between levels. As such, in coarser levels even the "long" displacements (in the original image) can be captured. Then, at each pyramid level, the correlation problem is initialized with the displacement field found in the previous one.

Furthermore, the affine transformation employed for removing the geometrical anisotropy accounts for the "flattening" of yarns. Indeed, the yarns crosssections are usually conceived as ellipses as a result of the manufacturing process. The motivation behind this anisotropy removal is that these regions do not provide much (relevant) information, especially for the aforementioned binary images. Thus, the isotropic version provides images that are smaller but with equivalent relevant information. It should be noted that this discussion is motivated by the fact that the current study is performed on specimens at the meso-scale. Moreover, the affinity employed for removing (or reducing) the anisotropy does not need to be exceptionally "precise", but rather approximate since the goal is to obtain in average more isotropic shapes. A similar argument 


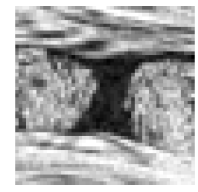

(a) $f$

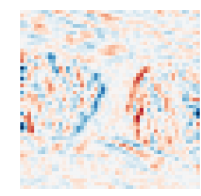

(d) $\nabla_{x}\{f\}$

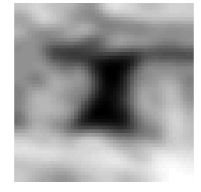

(b) $G \circledast f$

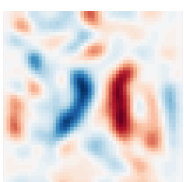

(e) $\nabla_{x}\{G \circledast f\}$

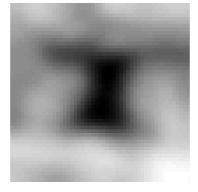

(c) $G \circledast G \circledast f$

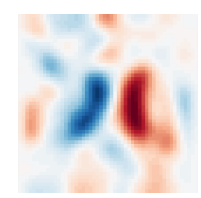

(f) $\nabla_{x}\{G \circledast G \circledast f\}$

Figure 6: An image $f$ recursively filtered by a Gaussian kernel $G$ displays horizontal gradients $\nabla_{x}$ that extend the range of "visibility" for correlation

can be made for textiles with more complex shapes. Whenever a systematic anisotropy (anisometry) is present, its reduction is beneficial [23].

Finally, a "complete" mechanical regularization technique [24] (based on the equilibrium gap [25-27]) is employed so as to better condition the problem. This favors displacement fields locally obeying a prescribed behavior (e.g., linear elasticity) in the bulk as well as in the surface of the studied samples. This strategy can be seen as a set of filters that locally dampen steep gradients and ensure smooth and differentiable fields.

\section{Discretization procedure}

The chosen intermediate representation for any descriptor is that of an image. As a consequence, a very convenient tool to evaluate differences between two textile representations is DVC (which deals with 3D images). As such, any of the previously descriptors needs to be projected into a regularly structured space. In other words, a discretization procedure is to be used so as to obtain voxels.

These voxels can be made meaningful by carefully choosing their values. 
A natural choice is to assign labels according to the structure they originally belonged to. This results in volumes with as many intensity levels as there are phases in the material. Since the present analysis considers only two phases, the obtained images are binary (or ternary if surrounding void is considered as a phase per se). It should be noted that this "translation" process does not result in any overall loss of information since it is always possible to retrieve the information in the original descriptors for any given voxel, and vice versa.

If the concerned descriptor is of topological nature, it needs to be converted into a geometrical one, this can be achieved via any of widely available textile pre-processors [28, 29]. Then, the transformation of the geometrical descriptors into images can be obtained via a FE mesh. In fact, the voxel-FE paradigm is appropriate for this case, it proposes to construct regular structured (uniform) meshes composed of cube elements [21]. Then each one of this FE elements can be interpreted as a voxel, thus creating an image.

These choices were taken into account in the development of a novel approach. The proposed alternative gracefully transitions from a continuous (parametric) description of the yarn towards a discretized (voxel) one. It is capable of handling the yarn path smoothing, the yarn discretization, and yarn interpenetrations under a flexible unified framework. Also, each one of these steps are easily implemented in parallel.

\subsection{Proposed approach}

First, the yarns are modeled as a collection of one-dimensional beam elements, these elements are constructed between every pair of consecutive control points. Then, a simple elastic behavior is applied on each yarn by means of the second-order differential Laplacian operator acting on the points coordinates. This effectively smoothens the yarn path and provides a "continuous" description of the yarn. As such, any point belonging to the $i$-th yarn centerline can be written as $\boldsymbol{r}_{i}(t)$ with $t \in[0,1]$ as the curvilinear coordinate along the yarn.

This strategy has the advantage of being easily adaptable to more complex behaviors (other than linear elasticity). In fact, if a better constitutive law is 
available (e.g., hyper- or hypo-elasticity $[18,30]$ ), it could be applied effortlessly. Additionally, this (already computed) Laplacian operator can be re-purposed for the reduction of control points. In fact, the reduced set of master points are easily identified as those having high Laplacian values. These will be the points of higher curvature, and thus necessary for describing the yarn path. An example of the Laplacian operator acting on a yarn is shown in 7 .

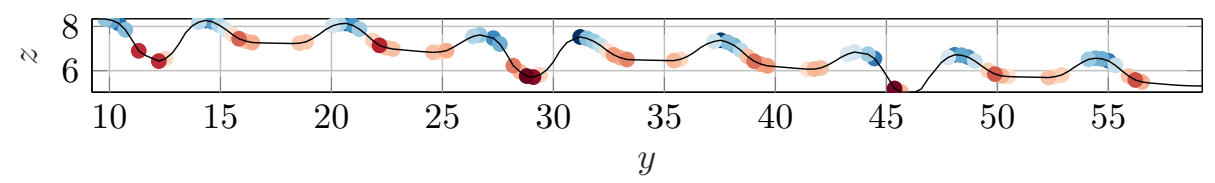

Figure 7: Laplacian operator acting on the coordinates of a yarn: the control points of higher curvature are automatically highlighted

Second, each yarn is discretized via its weighted distance function. This function determines the distance of a given point to the boundary (of the crosssection), it is maximum (100\%) for the points in the neutral fiber and decreases in value as the points approach the boundary where the distance attains its minimum $(0 \%)$. It is important to note that the function employs a weighted euclidean distance, with the weights being given by the aspect ratio of the ellipse cross-sections. Such distance function is computed for a grid of points (i.e., the voxel centers) regularly spaced (i.e., desired resolution). Additionally, any voxel placed outside of the yarn (i.e., negative distance) is discarded from the analysis. An example of such image is given in figure 8.

These operations can be expressed under a continuous formalism via the convolution operator

$$
I_{\mathcal{D}_{i}}(\boldsymbol{x})=\left(h_{\text {sign }} \circ d_{i}\right)\left(\boldsymbol{x}-\boldsymbol{r}_{i}\right) \circledast \delta\left(\boldsymbol{x}-\boldsymbol{r}_{i}\right)
$$

with $I_{\mathcal{D}_{i}}$ as the function containing all distances for the $i$-th yarn, $\delta\left(\boldsymbol{x}-\boldsymbol{r}_{i}\right)$ as its neutral fiber, $h_{\text {sign }}$ as the sign truncation function

$$
h_{\text {sign }}(s)= \begin{cases}1-s, & \text { for } s \leq 1 \\ 0, & \text { for } s<1\end{cases}
$$



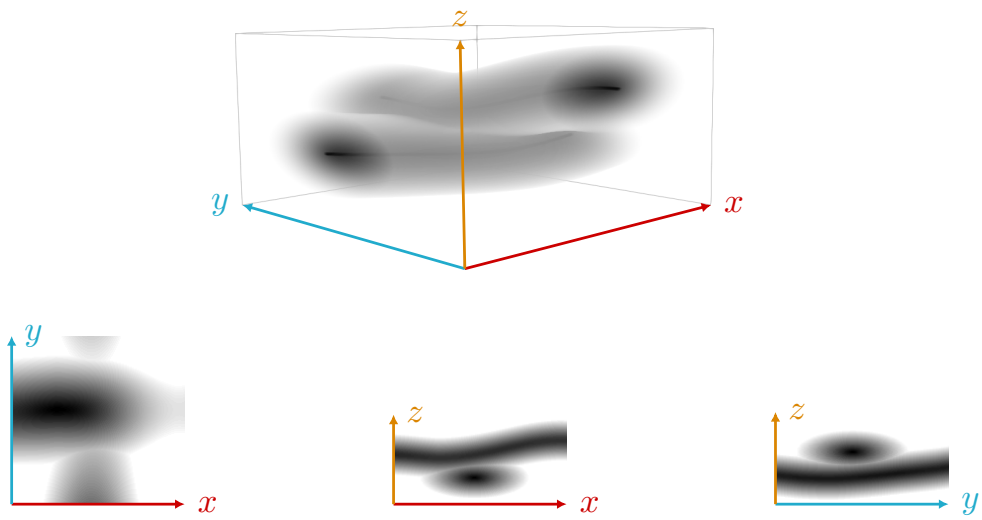

Figure 8: Different views for a sample image $I_{\mathcal{D}}$

and $d_{i}$ as the weighted distance function

$$
d_{i}(\{\boldsymbol{u}\})=\sqrt{\{\boldsymbol{u}\}^{\top}\left[\boldsymbol{C}_{i}\right]^{\top}\left[\boldsymbol{C}_{i}\right]\{\boldsymbol{u}\}}
$$

with the matrix $\left[\boldsymbol{C}_{i}\right]$ defining the cross-section shape for the $i$-th yarn. For example, in the case of an elliptical cross section aligned with the image axes, the diagonal elements of this matrix are given by the inverse of the ellipse radii.

Third, all considered voxels (with non-zero distances) for all yarns are "assembled" so as to form the complete image $I_{\mathcal{Y}}$, as show in figure 9. As such, all points considered only once are given the appropriate label (gray level) corresponding the yarn ID. Then, any voxel belonging to a region on yarn interpenetration (more than one yarn) will be assigned to the yarn to which it is "closest" (i.e., the one with greater $I_{\mathcal{D}}$ ). While simple, this handling of yarn interpenetration via the weighted distance function has proven extremely satisfactory.

Finally, the proposed approach outputs two volume images: a chart of distances $I_{\mathcal{D}}$ and a chart of yarns $I_{\mathcal{Y}}$. While the former contains floating point values contained between zero and one, the latter contains as many integer intensity values as there are yarns in the textile (plus the resin/air at zero).

Then, "thresholding" $I_{\mathcal{Y}}$ provides the aforementioned binary image $I_{\mathcal{B}}$. At this point, it is important to acknowledge that the image $I_{\mathcal{B}}$ can also be written 

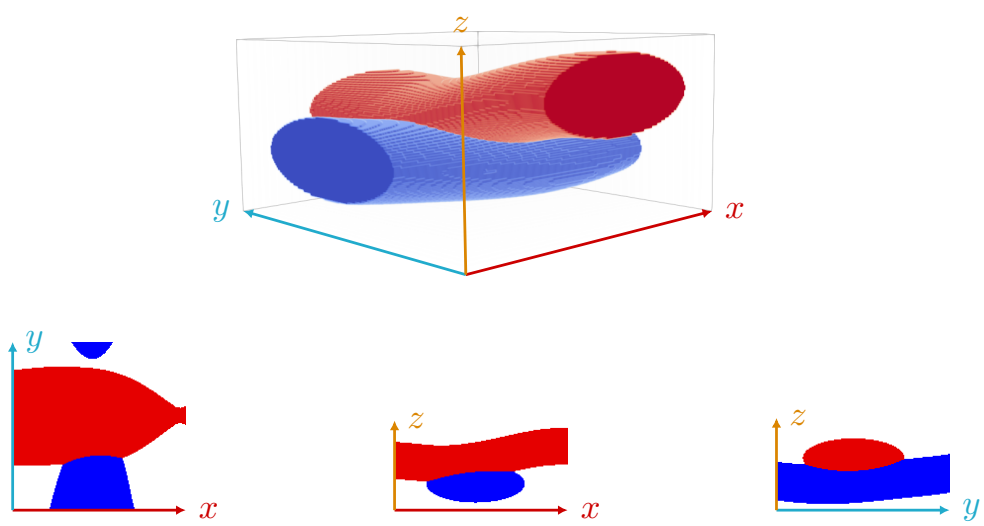

Figure 9: Different views for a sample image $I_{\mathcal{Y}}$

in a similar fashion as $I_{\mathcal{D}}$

$$
I_{\mathcal{B}_{i}}(\boldsymbol{x})=\left(h_{b i n} \circ d_{i}\right)\left(\boldsymbol{x}-\boldsymbol{r}_{i}\right) \circledast \delta\left(\boldsymbol{x}-\boldsymbol{r}_{i}\right)
$$

with $I_{\mathcal{B}_{i}}$ as the function containing the $i$-th yarn, and $h_{b i n}$ as the binary truncation function

$$
h_{b i n}(s)=\left(N_{1}-N_{0}\right) \cdot H(s-t)+N_{0}
$$

with the Heaviside function $H(\cdot)$, the desired intensity levels $N_{0}$ and $N_{1}$, and a threshold $0 \leq t<1$.

In the current context (tomography), the values of $N_{0}$ and $N_{1}$ represent the attenuation levels of the corresponding phases (i.e., the polymer resin and the carbon fiber yarns). An estimate of this values can be easily obtained from the histogram of a micro-CT image, which usually is bi-modal. The estimation of these values can be performed manually, or via a mixture model (e.g., Gaussian mixture model). It should be noted that, in the simplest scenario, they can simply take the values of $N_{0}=0$ and $N_{1}=1$. Then, it falls upon the correlation procedure to "adapt" these values by means of the intensity level corrections $v_{0}(\boldsymbol{x})$ and $v_{1}(\boldsymbol{x})$.

Similarly, the threshold parameter $t$ allows defining the distance (from the yarn centerline) at which the yarn surface is located. Hence, for $t=0$, the entire yarn is discretized; on the contrary, as $t$ tends to 1 , only the yarn centerline is 
considered.

Finally, such formulation has the added advantage of not needing to handle specifically overlapping yarns since the possibly superimposed yarn will provide the same value of $N_{1}$.

\subsection{Consequences for the registration procedure}

As it was previously introduced, the correlation strategy consists in "widening" the gradient lengths via filtering operations. This can be seen as a bank of Gaussian filters $G_{j}$ acting on the binary image $I_{\mathcal{B}}$.

$$
\begin{aligned}
\left\{I_{\mathcal{B}_{i}}(\boldsymbol{x})\right\} \circledast G_{j} & =\left\{\delta\left(\boldsymbol{x}-\boldsymbol{r}_{i}\right) \circledast\left(h_{b i n} \circ d_{i}\right)\left(\boldsymbol{x}-\boldsymbol{r}_{i}\right)\right\} \circledast G_{j} \\
& =\delta\left(\boldsymbol{x}-\boldsymbol{r}_{i}\right) \circledast\left\{\left(h_{b i n} \circ d_{i}\right)\left(\boldsymbol{x}-\boldsymbol{r}_{i}\right) \circledast G_{j}\right\} \\
& =\delta\left(\boldsymbol{x}-\boldsymbol{r}_{i}\right) \circledast \tilde{G}_{i j}
\end{aligned}
$$

with the kernel $\tilde{G}_{i j}$ as the custom $j$-th filter for the $i$-th yarn. This filter can be seen as a "wide" Gaussian filter acting on the yarn centerline.

Furthermore the pyramidal approach evoked earlier allows, through a mere recursion, obtaining any filter $G$ having a $2^{n}$ radius. This is coupled with an iterative subsampling of twice the blur radius.

\section{Setup}

Two sets of descriptors are available, each one of them will be employed in progressively more complex analyses.

\subsection{Available descriptors}

The first group consists in a tomographic scan and a geometrical descriptor of the same textile. This latter was extracted via a careful exploration of the $3 \mathrm{D}$ image, hence, it constitutes a good and close description of the observed sample. In the following, the image and the geometrical descriptor will be referred to as I1 and G1, respectively; they are illustrated in figure 10 .

The second group of descriptors is composed of two tomographic images and a FE mesh, all corresponding to the same textile. They will be referred 


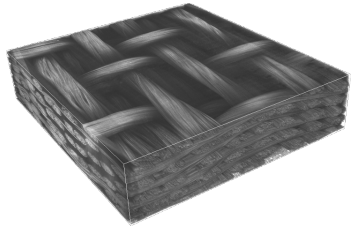

(a) I1

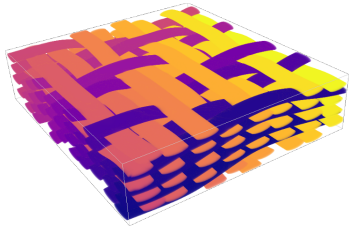

(b) G1

Figure 10: First group of textile descriptors: (a) a CT scan of a composite sample, and (b) its "equivalent" geometrical descriptor

to as I2, I3 and M1, accordingly. These observed samples I2 and I3 were used in a previous study. In reference [23], we have employed this pair for the identification of the so-called "metric differences" and "topological differences". Such study was capable of automatically detecting a voluntary alteration of sample I2: the removal of two yarns. The geometrical descriptor M1 can be expressed as the images $\mathrm{M} 1_{\mathcal{D}}, \mathrm{M} 1_{\mathcal{Y}}$ and $\mathrm{M} 1_{\mathcal{B}}$. They correspond to the different discretized representations discussed earlier (the distance functions, the yarns and the binary image), all three descriptors are shown in figure 11.

It is worth mentioning that the CT scans are obtained using classical reconstruction algorithms (FDK [31]) and under acquisition conditions that favors the differentiation of the different phases [23].

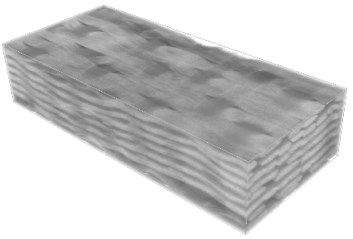

(a) I2

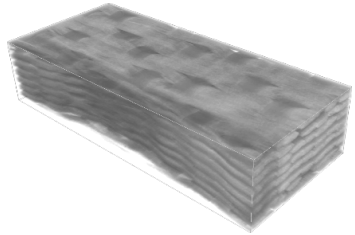

(b) I3

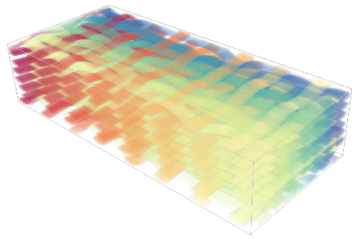

(c) M1

Figure 11: Second group of descriptors: (a) reference and (b) test samples observed through $\mathrm{CT}$, and (c) FE mesh with the same textile definition 


\subsection{Proposed analyses}

The first analysis is performed between the geometrical descriptor G1 and a numerically deformed version of itself, called G2 (i.e., model-model). This "arbitrary" deformation is obtained by applying a known Lagrangian displacement field $\boldsymbol{u}$ to G1. Such displacement was obtained from a previous study on known yarn deformations on this type of fabrics [32], so as to be representative of a plausible distortion. Then, the correlation procedure is performed as previously detailed with the descriptor G1 as reference, and ground truth is known for validating the result.

The second analysis is performed between the geometrical descriptor G1 and the image I1 (i.e., model-real). As previously stated, the descriptor was built from the image in question. Then, this correlation analysis should allow aligning the observed sample to the reference model G1.

The next group of analyses is performed in three steps. First, the pair of tomographic volumes I2 and I3 is analyzed [23]. Next, the pair of descriptors I2 and M1 is analyzed in a similar fashion as previously. In both cases the sample I2 is chosen as the reference configuration and both remaining descriptors are used as the test (deformed) configurations in each step. These third and fourth analyses are analogous to the first two, but implemented on a different set of descriptors. The final fifth step integrates both previous results (i.e., combined analysis).

A schematic representation of the proposed analyses is shown in figure 12 


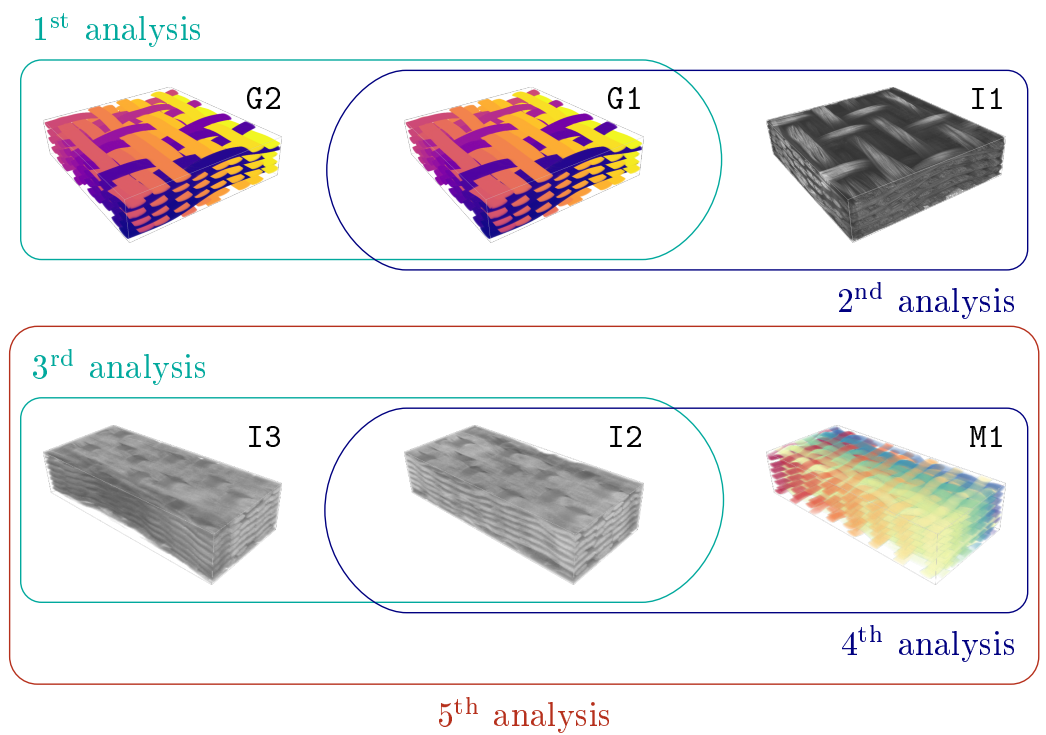

Figure 12: Proposed sets of analyses combining the available descriptors

\section{Results}

\subsection{Model-model registration results}

The different pyramid levels can be seen in figures 13 and 14. For visualization purposes, the warp and weft yarns were assigned different gray levels. As it can be seen, this strategy does help in widening structures boundaries or interfaces (i.e., the image gradients).

The correlation procedure is performed using 6885 kinematic degrees of freedom represented using cube elements with length $16 \mathrm{px}$. This length approximates the radius of the circular cross-section of the yarns (in the isotropic space).

A good agreement can be observed between the imposed and measured displacement fields, as shown in figure 15. Furthermore, the image of residuals, testifies of a good alignment between both virtual models, as seen in figure 16 . These results are encouraging: correlation performs extremely well in spite of a complex deformation, a poor texture, and a relatively poor chosen kinematics. 


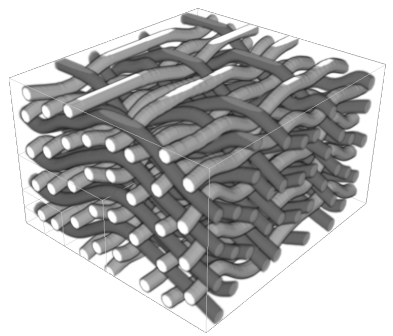

(a) Pyramid level 1

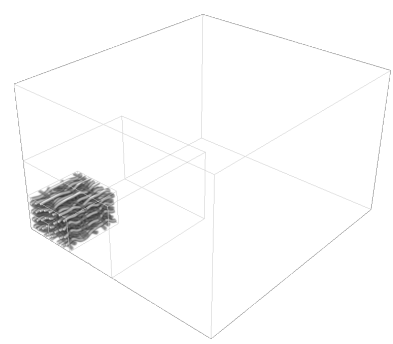

(c) Pyramid level 3

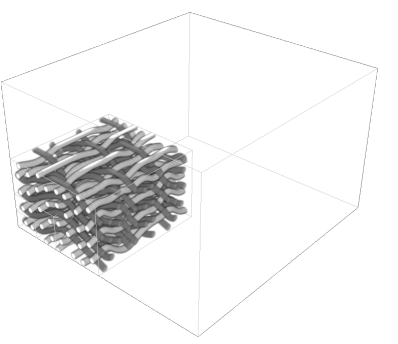

(b) Pyramid level 2

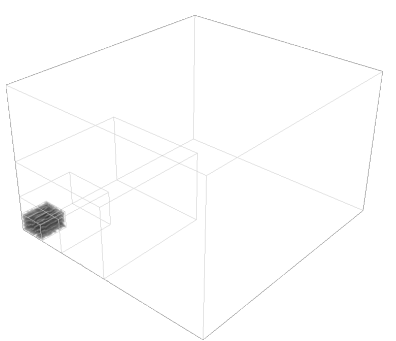

(d) Pyramid level 4

Figure 13: Volume representation of the different pyramid levels for G1

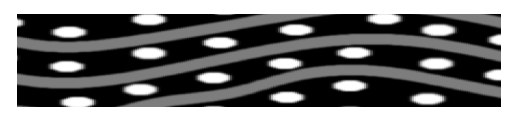

(a) Pyramid level 1

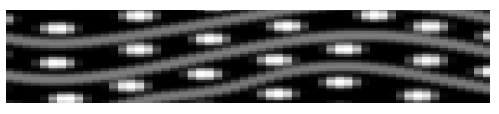

(c) Pyramid level 3

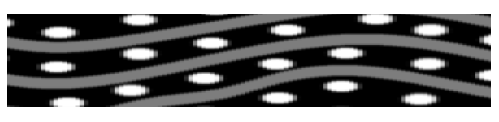

(b) Pyramid level 2

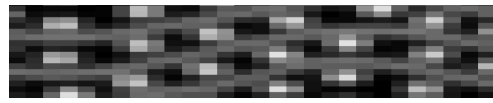

(d) Pyramid level 4

Figure 14: $(x-z)$ mid-plane representation of the different pyramid levels for G1 


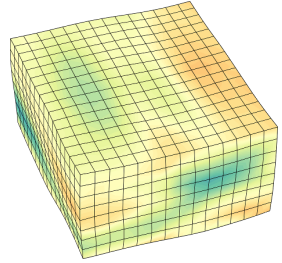

(a) $u_{x}$

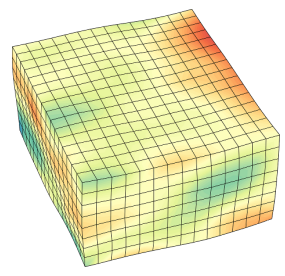

(d) $u_{x}$

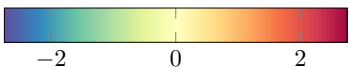

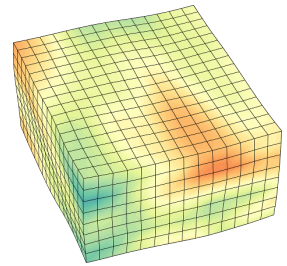

(b) $u_{y}$

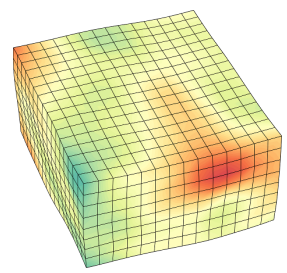

(e) $u_{y}$

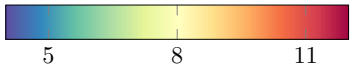

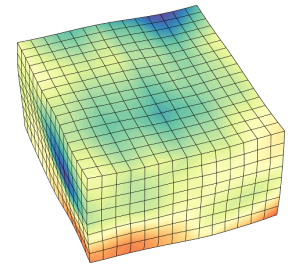

(c) $u_{z}$

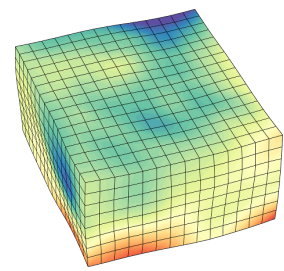

(f) $u_{z}$

Figure 15: Components of the displacement fields for G1 and G2: (a)-(c) known field, and (d)-(f) measured field (in px)

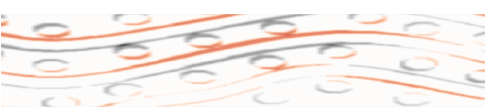

(a) $(x-z)$ mid-plane

(c) $(x-z)$ mid-plane

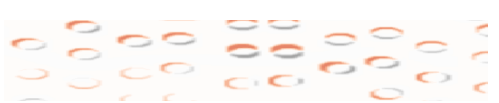

(b) $(y-z)$ mid-plane

(d) $(y-z)$ mid-plane

Figure 16: Different mid-planes for the residuals for G1 and G2: (a)-(b) before and (c)-(d) after correlation 


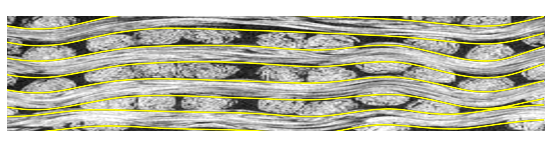

(a) I1 $(\boldsymbol{x})$

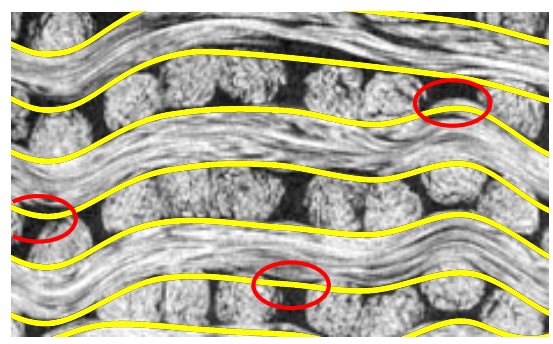

(c) $\operatorname{I1}(\boldsymbol{x})$

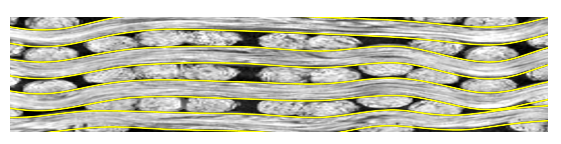

(b) $\mathrm{I} 1(\boldsymbol{x}+\boldsymbol{u}(\boldsymbol{x}))$

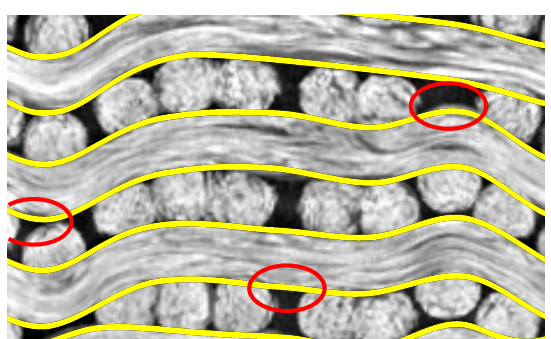

(d) I1 $(\boldsymbol{x}+\boldsymbol{u}(\boldsymbol{x}))$

Figure 17: $(x-z)$ mid-plane of I1 before and after correlation, the model G1 is shown with outlined yarn borders, the ellipses highlight some regions of improved alignment; for visualization purposes the images in (a) and (b) are "zoomed" and shown in the isotropic configuration in (c) and (d) respectively

\subsection{Model-real registration results}

The correlation results are analyzed by comparing the reference model G1 with the sample I1. This is achieved by superimposing the yarn boundaries (as described by the model) on top of the sample in the "test" configuration I1 $(\boldsymbol{x})$ and the sample in the "corrected" configuration I1 $(\boldsymbol{x}+\boldsymbol{u}(\boldsymbol{x}))$, as shown in figure 17. These images showcase a slight improvement over the already good alignment between both descriptors. Some regions in which the tomographic image is "unwrapped" so as to better fit the model are highlighted.

Furthermore, thanks to the relaxed brightness conservation formulation, the intensity values of the micro-CT image are also "corrected". This translates into yarns with more ellipsoid cross-sections and a better contrast between phases, as imposed by the binary image issued from the model G1. As figure 18 shows, the improved separation of classes becomes clear in the corresponding histogram for the image I1, where its bi-modal nature becomes more pronounced. 


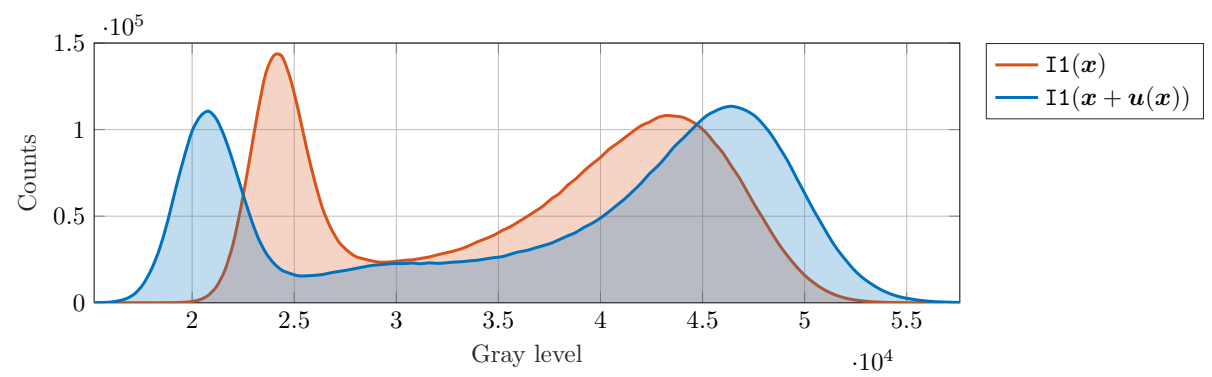

Figure 18: Histograms before and after correlation for the micro-CT image

\subsection{Combined results}

This final collection of results will present a three-part analysis.

The results for the first step are the so-called "metric differences" and "topological differences" between of both samples [23]. On the one hand, the "metric differences" inform on the relative strains $\boldsymbol{\epsilon}$ between the observed textiles I2 and I3, as obtained from the measured displacement field $\boldsymbol{u}_{3}(\boldsymbol{x})$. The trace of this relative strain tensor (i.e., an invariant reflecting the local change in volume), shown in figure 19a, is localized around the missing yarns (due to a re-arrangement of the remaining yarns). On the other hand, the "topological differences" highlight the missing yarns (they break the assumption of conserved topology). They are obtained by means of the semi-residual due to displacement only, which does not account for the brightness and contrast corrections, as shown in figure 19b. It is important to note that the registration is performed correctly despite the sizable "topological differences" induced by the missing yarns.

The second step consists in adapting the (theoretical) model M1 onto the sample I2. The necessary intermediate representation of M1 as a binary image is shown in figure 20a. After registration, the found displacement field is applied onto the corresponding geometrical descriptor so as to obtain an "aligned" version of M1. This result, shown in figure 20b, leads to the "segmentation" and identification of yarns.

Finally, the results of the two preceding calculations are combined. More 


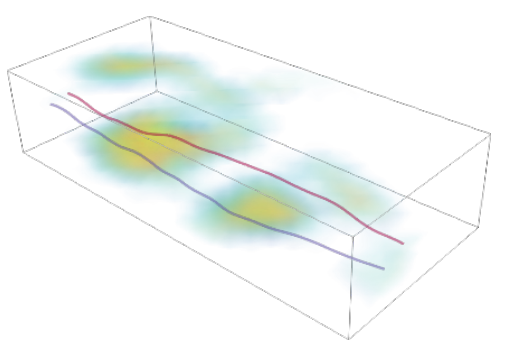

(a) Trace of the relative strain tensor

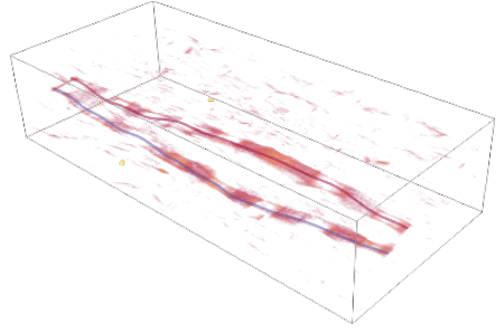

(b) Semi-residual due to displacement

only

Figure 19: The signature of the missing yarns is clear in (a) the "metric differences", as well as in (b) the "topological differences" between the analyzed volumes I2 and I3

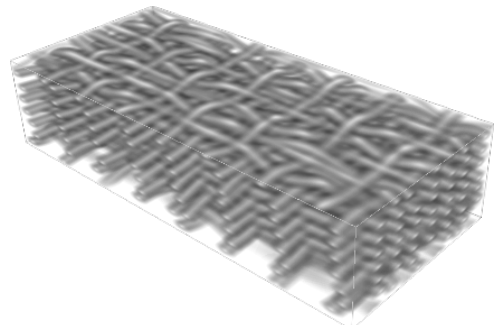

(a) $\operatorname{M} 1_{\mathcal{B}}(\boldsymbol{x})$

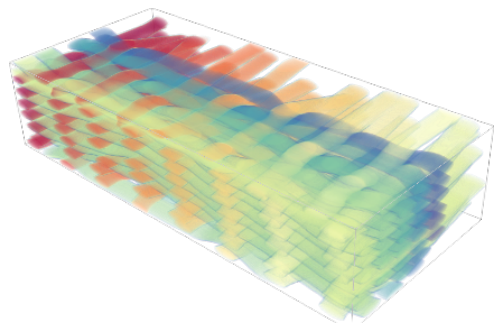

(b) $\operatorname{M} 1 \mathcal{Y}\left(\boldsymbol{x}+\boldsymbol{u}_{4}(\boldsymbol{x})\right)$

Figure 20: The different representations of M1: (a) as a (discretized) binary image, and (b) as a model aligned with the reference sample.

precisely, the "topological differences" from I2 and I3, and the "aligned" model from I2 and M1. Since all results are expressed under the same reference configuration, they can be swiftly compared.

As such, it is possible to query the "amount" of topological differences per yarn

$$
C_{y}=\sum_{\boldsymbol{x} \in \mathcal{N}_{y}} \mathrm{M}_{\mathcal{B}}\left(\boldsymbol{x}+\boldsymbol{u}_{4}(\boldsymbol{x})\right) \cdot\left(\mathrm{I} 3\left(\boldsymbol{x}+\boldsymbol{u}_{3}(\boldsymbol{x})\right)-\mathrm{I} 2(\boldsymbol{x})\right)
$$

where $\mathcal{N}_{y}$ represents the (neighborhood of) voxels that correspond the $y$-th yarn

$$
\mathcal{N}_{y}=\left\{\boldsymbol{x} \mid \operatorname{M1} \mathcal{Y}\left(\boldsymbol{x}+\boldsymbol{u}_{4}(\boldsymbol{x})\right)=y\right\}
$$


From this tally $C_{y}$, shown in figure 21a, two yarns are clearly distinguished from the rest. A new virtual model which only considers these yarns is shown in figure 21b. Clearly, these yarns correspond to the missing ones, which (in this particular ordering) correspond to the yarns with ID numbers 20 and 26. These ID numbers are extremely relevant because (if the weaving patter is known) it is possible to identify their role in the textile architecture.

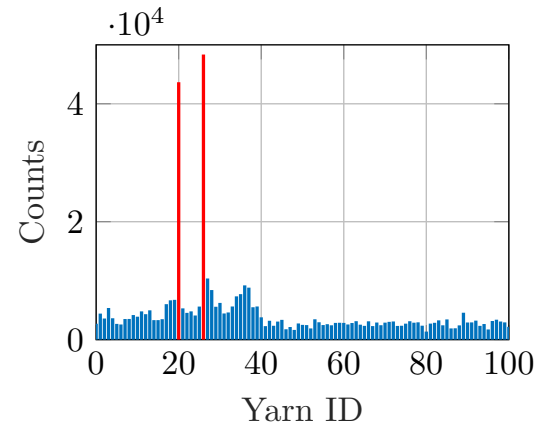

(a)

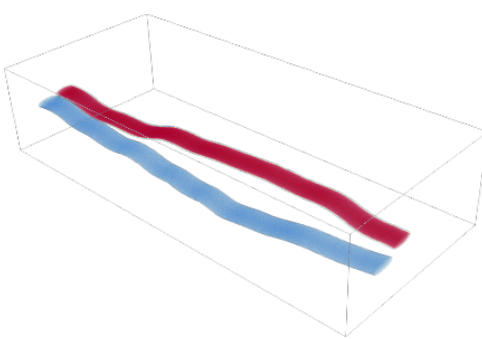

(b)

Figure 21: (a) The amount of topological differences per yarn helps identify (b) the missing yarns.

At this point, the obtained results can be further exploited if a precise segmentation of the yarns is desired. Since the goal of this study is to "align the yarns", the kinematic decomposition (i.e., the degrees of freedom) is not detailed enough so as to precisely (i.e., voxelwise) conform to the yarn boundaries (otherwise, it would lead to ill-conditioning), and as such the proposed registration is not by itself a substitute to segmentation.

However, it does offer an excellent "initialization" for appropriate segmentation techniques $[33,34]$, and only minor adjustments of boundaries remain to be determined. In such sense, one could image employing algorithms based on graphs [35, 36], on some form of energy minimization [37], on some a priori information [38] or, even better, based a combination of all of those [39].

For example, the alignment of the model to the sample provides sufficient seeds (e.g., the yarn centerlines) for correctly segmenting the yarns in the CT 
image. This helps to overcome the challenge faced when, two (or more) yarns are so heavily compressed against each other, that they become visually indistinguishable (at the meso-scale). Alternatively, the yarns could be formulated as "attractors" (or "repulsors") that would minimize (or maximize) a certain energy functional.

Finally, the proposed correlation framework represents a novel paradigm for the "segmentation" of yarns. Indeed, this method presents itself as a topdown approach, much unlike most "classical" bottom-up approaches. The latter bottom-up methods start from the smallest feature of interest (i.e., the intensities at each voxel) in order to "build-up" towards a sought structure (e.g., the yarns, the textile). Such process is mostly of local nature (i.e., the yarns) and the notion of a textile is faint. On the contrary, the proposed top-down method starts with the global notion of a textile model, which is then aligned towards a given descriptor. This constitutes a major advantage of the technique since it is capable of robustly handling local discrepancies thanks to the information provided elsewhere by the (global) model. This is to be contrasted with most segmentation approaches based on local features, that may suffer from local indeterminations leading to topological violations (such as wrong pairing of yarn segments).

\section{Conclusions}

The use of Digital Volume Correlation for the dialog between various textile descriptors was presented. This technique, applied to 3D woven composites, is based on the key concept of the conservation of topology. The robustness of this approach comes from the use of a reference configuration that provides the algorithm with a vast amount of information with a considerably low effort.

Different scenarios involving different descriptors were explored, and by combining the different steps, an automatic nondestructive testing (NDT) procedure for $3 \mathrm{D}$ woven composites was achieved. It should be noted that the detection of missing yarns (or any other anomaly) was not the intended target of the 
analysis. However, given that they do not conform to the reference or to the assumption of constant topology, they appeared naturally. In addition, these missing yarns were identified (i.e., by ID number) thanks to the relationships established with a model of the textile.

This technique allows the use of a single topological descriptor for the many different analyses that are involved in the study of the woven composites. As such, it has many applications on different fields.

For example, in the domain of mechanical simulation and validation of forming models using architectures extracted from micro-CT images [40, 41]. Usually, these techniques call for a meshing procedure per each analyzed sample. This results in as many meshes as samples, which in turn, encumbers the comparative analysis between the results of these many simulations. However, if only one topological descriptor is considered, only one mesh and potentially only one (base/reference) simulation would be needed. The mesh could simply be adapted to each new sample, as shown in the examples here with the geometrical descriptors and the tomographic images. Even more interestingly, the reference simulation could be "adapted" to the new "deformed" sample because the displacement field relating both configurations is known. Evidently, this form of transfer learning necessitates an appropriate description of the phenomena to be studied. Some simulations have been planned with this purpose.

Additionally, the technique can be used as a tool for non-destructive testing: it can be performed either on the "metric differences", on the "topological differences", or on both. The former could inform on "typical" modes of deformation, which would allow defining novel and appropriate metrics [42]. These could then enable the identification of anomalous strain patterns. Clearly, this implies a multitude of comparative analyses on multiple woven composite samples so as to build a "proper" database of such modes. Similarly, the "topological differences" could be analyzed for the identification of patterns or structures that would indicate weaving anomalies using machine learning techniques.

Furthermore, it should be noted that the Gaussian filter employed alongside the discretization procedure for descriptors other than images leads to a mod- 
erate "loss of information" (since it erases high frequency features). The most important property of this filter is that even if the synthetic image generated by the model is too sharp or too contrasted, the mollifying nature of the Gaussian filter will bring both images of interest (the reconstructed CT scan and the synthetic image) closer together.

Moreover, these operations (discretization and convolution) occur only once: before the correlation procedure. That means that they are not updated, unlike the displacement field which does evolve during the iterations. This could be considered as a limitation of the current proposition since the convolution and the distortion of the fabric do not commute. As such, a good registration may not be possible for cases of extreme distortions. For such a reason, it is proposed to alternate between both operations during iterations. That is, to apply the estimated displacement to the original descriptor, filter the discretized image, and determine the additional correction to the displacement field; as is done with multi-modality registration [43]. Repeating this operation iteratively would permit a better estimation of the correction fields. In fact, the proposed discretization procedure aligns perfectly with this goal. The approach is currently being explored.

This proposed extension to the technique could also have an impact on the discretization and convolution operations. The construction of the binary image from the model involves the definition of the binary levels which could be progressively adapted by using smooth indicator functions blurred along the fiber direction [44]. Similarly, the parameters for the convolution operation (Gaussian radii) could become additional degrees of freedom (besides displacement and intensity levels). As such, the yarns would be modeled after their yarn centerline locally filtered by the appropriate kernel. Such approach was alluded to when discussing the consequences of registration in the discretization procedure (i.e., a "wide" Gaussian filter) [45].

Finally, this technique could also benefit from the inherent periodicity of the fabric and further integrate elements so as increase its efficiency. 


\section{Acknowledgements}

This work is supported by the PRC MECACOMP, project co-founded by DGAC and Safran group, involving Safran group companies, ONERA, CNRS and other academic partners. AMQ acknowledges the support of a $\mathrm{PhD}$ grant from ANRT and Safran Tech.

\section{References}

[1] S. Nehme, A. Hallal, F. Fardoun, R. Younes, B. Hagege, Z. Aboura, M. Benzeggagh, F. H. Chehade, Numerical/analytical methods to evaluate the mechanical behavior of interlock composites, Journal of Composite Materials 45 (2011) 1699-1716. doi:10.1177/0021998310385592.

[2] F. Desplentere, S. V. Lomov, D. L. Woerdeman, I. Verpoest, M. Wevers, A. Bogdanovich, Micro-CT characterization of variability in 3D textile architecture, Composites Science and Technology 65 (2005) 1920-1930. doi:10.1016/j.compscitech.2005.04.008.

[3] N. Naouar, E. Vidal-Salle, J. Schneider, E. Maire, P. Boisse, 3D composite reinforcement meso F.E. analyses based on X-ray computed tomography, Composite Structures 132 (2015) 1094-1104. doi:10.1016/j. compstruct.2015.07.005.

[4] D. J. Bull, L. Helfen, I. Sinclair, S. M. Spearing, T. Baumbach, A comparison of multi-scale 3D X-ray tomographic inspection techniques for assessing carbon fibre composite impact damage, Composites Science and Technology 75 (2013) 55-61. doi:10.1016/j.compscitech.2012.12.006.

[5] R. de Luis-García, R. Deriche, C. Alberola-López, Texture and color segmentation based on the combined use of the structure tensor and the image components, Signal Processing 88 (2008) 776-795. doi:10.1016/j.sigpro. 2007.09.019. 
[6] O. C. Zienkiewicz, The finite element method, volume 3, McGraw-hill London, 1977.

[7] M. Sherburn, Geometric and Mechanical Modelling of Textiles, Ph.D. thesis, University of Nottingham, 2007. doi:10.1016/j.compositesa.2009.04.009.

[8] R. Gras, H. Leclerc, F. Hild, S. Roux, J. Schneider, Identification of a set of macroscopic elastic parameters in a 3D woven composite: Uncertainty analysis and regularization, International Journal of Solids and Structures 55 (2015) 2-16. doi:10.1016/j.ijsolstr.2013.12.023.

[9] I. Verpoest, S. V. Lomov, Virtual textile composites software WiseTex: Integration with micro-mechanical, permeability and structural analysis, Composites Science and Technology 65 (2005) 2563-2574. doi:10.1016/j. compscitech.2005.05.031.

[10] B. K. Bay, Methods and applications of digital volume correlation, The Journal of Strain Analysis for Engineering Design 43 (2008) 745-760. doi: 10.1243/03093247JSA436.

[11] H. Leclerc, J. N. Périé, S. Roux, F. Hild, Voxel-Scale Digital Volume Correlation, Experimental Mechanics 51 (2011) 479-490. doi:10.1007/ s11340-010-9407-6.

[12] A. Mendoza, S. Roux, J. Schneider, E. Parra, E. Obert, Unwrapping Textile Fabric, in: 3rd International Conference on Tomography of Materials and Structures, 2017, pp. 1-2.

[13] A. Mendoza, J. Schneider, E. Parra, E. Obert, S. Roux, Bridging the gap between modeling and analysis for 3D woven composites using Digital Volume Correlation, in: 7th International Symposium on Aircraft Materials (ACMA2018), 2018.

[14] A. Mendoza, J. Schneider, E. Parra, E. Obert, S. Roux, Bridging the gap between modeling and analysis for 3D woven composites using Digital Vol- 
ume Correlation, in: ECCM18 - 18 th European Conference on Composite Materials, 2018.

[15] S. V. Lomov, D. S. Ivanov, I. Verpoest, M. Zako, T. Kurashiki, H. Nakai, S. Hirosawa, Meso-FE modelling of textile composites: Road map, data flow and algorithms, Composites Science and Technology 67 (2007) 18701891. doi:10.1016/j.compscitech.2006.10.017.

[16] M. Ansar, W. Xinwei, Z. Chouwei, Modeling strategies of 3D woven composites: A review, Composite Structures 93 (2011) 1947-1963. doi: 10.1016/j.compstruct.2011.03.010.

[17] D. Durville, Numerical simulation of entangled materials mechanical properties, Journal of Materials Science 40 (2005) 5941-5948. doi:10.1007/ s10853-005-5061-2.

[18] N. Naouar, E. Vidal-Sallé, J. Schneider, E. Maire, P. Boisse, Meso-scale $\mathrm{FE}$ analyses of textile composite reinforcement deformation based on $\mathrm{X}$ ray computed tomography, Composite Structures 116 (2014) 165-176. doi: 10.1016/j.compstruct.2014.04.026.

[19] S. V. Lomov, Modelling the geometry of textile composite reinforcement, 2010.

[20] F. Stig, S. Hallström, Spatial modelling of 3D-woven textiles, Composite Structures 94 (2012) 1495-1502. doi:10.1016/j.compstruct.2011.12.003.

[21] G. Hello, J. Schneider, Z. Aboura, Numerical Simulations of Woven Composite Materials With Voxel-FE Models, in: 16th European Conference on Composite Materials (ECCM 2014), June, 2014, pp. 22-26.

[22] B. K. Bay, T. S. Smith, D. P. Fyhrie, M. Saad, Digital volume correlation: Three-dimensional strain mapping using X-ray tomography, Experimental Mechanics 39 (1999) 217-226. doi:10.1007/BF02323555. 
[23] A. Mendoza, J. Schneider, E. Parra, E. Obert, S. Roux, Differentiating 3D textile composites: A novel field of application for Digital Volume Correlation, Composite Structures 208 (2019) 735-743. doi:10.1016/j.compstruct. 2018.10.008.

[24] A. Mendoza, J. Neggers, F. Hild, S. Roux, Complete mechanical regularization applied to digital image and volume correlation, Computer Methods in Applied Mechanics and Engineering 355 (2019) 27-43. doi: 10.1016/j.cma.2019.06.005.

[25] D. Claire, F. Hild, S. Roux, A finite element formulation to identify damage fields: the equilibrium gap method, International Journal for Numerical Methods in Engineering 61 (2004) 189-208.

[26] S. Roux, F. Hild, H. Leclerc, Mechanical assistance to DIC, Procedia IUTAM 4 (2012) 159-168. doi:10.1016/j.piutam.2012.05.018.

[27] Z. Tomicevic, F. Hild, S. Roux, Mechanics-Aided Digital Image Correlation, The Journal of Strain Analysis for Engineering Design 48 (2013) 330-343.

[28] S. V. Lomov, A. V. Gusakov, G. Huysmans, A. Prodromou, I. Verpoest, Textile geometry preprocessor for meso-mechanical models of woven composites, Composites Science and Technology 60 (2000) 2083-2095. doi: 10.1016/S0266-3538(00)00121-4.

[29] H. Lin, L. P. Brown, A. C. Long, Modelling and simulating textile structures using TexGen, in: Advanced Materials Research, volume 331, Trans Tech Publ, 2011, pp. 44-47.

[30] M. A. Khan, T. Mabrouki, E. Vidal-Sallé, P. Boisse, Numerical and experimental analyses of woven composite reinforcement forming using a hypoelastic behaviour. Application to the double dome benchmark, Journal of Materials Processing Technology 210 (2010) 378-388. doi:10.1016/j. jmatprotec.2009.09.027. 
[31] L. A. Feldkamp, L. C. Davis, J. W. Kress, Practical cone-beam algorithm, J Opt Soc Am 1 (1984) 612-619. doi:10.1364/JOSAA.1.000612.

[32] A. Mendoza, J. Schneider, E. Parra, S. Roux, Measuring yarn deformations induced by the manufacturing process of woven composites, Composites Part A: Applied Science and Manufacturing 120 (2019) 127-139. doi:10. 1016/j.compositesa.2019.02.008.

[33] M. E. Leventon, W. E. L. Grimson, O. Faugeras, Statistical shape influence in geodesic active contours, in: Proceedings of the IEEE Computer Society Conference on Computer Vision and Pattern Recognition, volume 1, IEEE, 2000, pp. 316-323. doi:10.1109/CVPR.2000.855835.

[34] S. Beucher, F. Meyer, The morphological approach to segmentation: the watershed transformation, Optical Engineering-New York-Marcel Dekker Incorporated- 34 (1992) 433-433.

[35] Z. J. Jie, Graph cuts segmentation with geometric shape priors for medical images, in: Proceedings of the IEEE Southwest Symposium on Image Analysis and Interpretation, IEEE, 2008, pp. 109-112. doi:10.1109/SSIAI. 2008.4512297.

[36] Y. Boykov, O. Veksler, R. Zabih, Fast Approximate Energy Minimization via Graph Cuts, IEEE Transactions on pattern analysis and machine intelligence 23 (2001) 122-1239.

[37] T. F. Cootes, C. J. Taylor, D. H. Cooper, J. Graham, Active shape models their training and application, Computer Vision and Image Understanding 61 (1995) 38-59. doi:10.1006/cviu.1995.1004.

[38] A. Mendoza Quispe, C. Petitjean, Shape prior based image segmentation using manifold learning, in: 5th International Conference on Image Processing, Theory, Tools and Applications 2015, IPTA 2015, 2015. doi: 10.1109/IPTA.2015.7367113. 
[39] C. Couprie, L. Grady, L. Najman, H. Talbot, Power watershed: A unifying graph-based optimization framework, IEEE Transactions on Pattern Analysis and Machine Intelligence 33 (2011) 1384-1399. doi:10.1109/TPAMI. 2010.200 .

[40] N. Naouar, E. Vidal-Salle, E. Maire, J. Schneider, P. Boisse, Meso F.E. simulation of composite reinforcement deformation based on X-ray computed tomography, Materiaux \& Techniques 104 (2014) 165-176. doi: $10.1051 / \mathrm{mattech} / 2016029$.

[41] Z. Yousaf, P. Potluri, P. J. Withers, D. Mollenhauer, E. Zhou, S. Duning, Digital element simulation of aligned tows during compaction validated by computed tomography (CT), International Journal of Solids and Structures (2016). doi:10.1016/j.ijsolstr.2017.05.044.

[42] R. de Luis-García, C. Alberola-López, C. F. Westin, On the choice of a tensor distance for DTI white matter segmentation, in: Mathematics and Visualization, 202519, Springer, Berlin, Heidelberg, 2012, pp. 283-306. doi:10.1007/978-3-642-27343-8\{_\}15.

[43] A. Charbal, J. E. Dufour, F. Hild, M. Poncelet, L. Vincent, S. Roux, Hybrid Stereocorrelation Using Infrared and Visible Light Cameras, Experimental Mechanics 56 (2016) 845-860. doi:10.1007/s11340-016-0127-4.

[44] D. Wassermann, L. Bloy, E. Kanterakis, R. Verma, R. Deriche, Unsupervised white matter fiber clustering and tract probability map generation: Applications of a Gaussian process framework for white matter fibers, NeuroImage 51 (2010) 228-241. doi:10.1016/j.neuroimage.2010.01.004.

[45] A. Charbal, J. E. Dufour, A. Guery, F. Hild, S. Roux, L. Vincent, M. Poncelet, Integrated Digital Image Correlation considering gray level and blur variations: Application to distortion measurements of IR camera, Optics and Lasers in Engineering 78 (2016) 75-85. doi:10.1016/j.optlaseng.2015. 09.011. 